\title{
The Epidemiology and Outcomes of Mental Disorders in Critically III Patients With Systemic Lupus Erythematosus: A Population-Based Study
}

\author{
Lavi Oud
}

\begin{abstract}
Background: Hospitalized patients with systemic lupus erythematosus (SLE) often require critical care, and SLE is the most common autoimmune disease in the intensive care unit (ICU). Mental disorders are highly prevalent among patients with SLE and are associated with increased morbidity and premature death in this population. However, the association of mental disorders with ICU utilization among patients with SLE and their prognostic impact among those admitted to ICU is unknown.
\end{abstract}

Methods: We performed a retrospective cohort study, using the Texas Inpatient Public Use Data File to identify SLE hospitalizations aged $\geq 18$ years during 2009 - 2014. Mental disorders were defined by the taxonomy of the Healthcare Cost and Utilization Project's Clinical Classification Software Category 5. The patterns of ICU admission among SLE hospitalizations with and without mental disorders were examined. Multivariable logistic regression modeling was used to examine the association of mental disorders and short-term mortality (defined as hospital death or discharge to hospice) among ICU admissions.

Results: Among 94,338 SLE hospitalizations 35,793 (37.9\%) had mental disorders. There was no difference in the rates of ICU admission among SLE hospitalizations with and without mental disorders ( $37 \%$ vs. $37.2 \%$, respectively; $\mathrm{P}=0.5999)$, and similar rates of mental disorders were found among SLE hospitalizations with and without ICU admission ( $37.8 \%$ vs. $38 \%$, respectively; $\mathrm{P}=0.5408$ ). The volume of SLE ICU admissions with and without mental disorders rose between 2009 and 2014 by $60.3 \%$ vs. $7.9 \%$, respectively. When compared to those without mental disorders, SLE ICU admissions with mental disorders were older (age $\geq 65$ years, $23.6 \%$ vs. $21.4 \%$, respectively) and had higher burden of comorbid conditions. Unadjusted short-term mortality among SLE ICU admissions with and without mental disorders was $4.8 \%$ and $5.8 \%$, respectively and

Manuscript submitted June 19, 2020, accepted July 3, 2020

Published online July 22, 2020

Division of Pulmonary and Critical Care Medicine, Department of Internal Medicine, Texas Tech University Health Sciences Center at the Permian Basin, Odessa, TX 79763, USA. Email: lavi.oud@ttuhsc.edu

doi: https://doi.org/10.14740/jocmr4269 mental disorders were associated with lower short-term mortality on adjusted analyses (adjusted odds ratio (aOR): 0.826; 95\% confidence interval (CI): 0.734 - 0.930).

Conclusions: There was no difference in the frequency of mental disorders among hospitalized patients with SLE with and without ICU admission. However, the growth in the volume of ICU admissions with SLE over time involved predominantly patients with mental disorders. Among ICU admissions, mental disorders were associated with lower short-term mortality.

Keywords: Systemic lupus erythematosus; Critical illness; Mental disorders; Intensive care unit; Mortality

\section{Introduction}

Systemic lupus erythematosus (SLE) is a multisystem autoimmune disease of an unknown etiology [1]. Although the longterm survival of SLE has progressively improved over the past decades [2], the risk of premature death has plateaued since 1999 [3] and patients continue to be affected by considerable morbidity [4]. Patients with SLE are commonly hospitalized [5] and SLE has become over the past 2 decades the most common autoimmune disease in the intensive care unit (ICU) [6] with reported high risk of death $[7,8]$.

Mental disorders are markedly more common among patients with SLE than in the general population [9] and are associated with increased incidence of cardiovascular disease, physical disability and premature death [10]. The mechanisms modulating the association of mental disorders with adverse outcomes in SLE have not been elucidated. However, some potentially explanatory data were described in the general population, where mental disorders are well-documented to be associated with increased burden of medical comorbidities and [11] and substantially reduced long-term survival [12]. It appears that the increased morbidity and mortality among patients with mental disorders in the general population is multifactorial, including prevalent risky lifestyle choices, altered engagement with the health care system, adverse effects of psychotropic medications, and poorer quality of preventive and interventional medical care [13]. Thus, it may be pos- 
tulated that mental disorders may increase the risk of acute health crises among patients with SLE, including need for ICU admission and may lead in turn to worse outcomes of the critically ill. However, there have been no studies, to our knowledge, on mental disorders among critically ill patients with SLE, while only scarce data are available on the epidemiology and outcomes of critically ill patients with mental disorders in the general population.

A recent study documented higher rates of mental disorders among critically ill mechanically ventilated patients, as compared to other hospitalized patients and the general population in Denmark [14]. These findings prompted the investigators to hypothesize that mental disorders may predispose patients to critical illness [14]. Mental disorders were reported in $6.2 \%$ [14] to $36.4 \%$ [15] of ICU patients in the general population and studies on the association between mental disorders and short-term mortality showed either no difference [16] or increased risk of death [17], as compared to those without mental disorders.

Data on the epidemiology and outcomes of mental disorders among critically ill patients with SLE can inform critical care resource allocation, preventive and therapeutic efforts in the general patient care, clinicians' prognostic decision-making during critical illness, and future performance improvement initiatives.

Here, we report an examination of a population-based cohort of hospitalized patients with SLE with two aims: 1) characterize patterns of ICU utilization among patients with mental disorders; 2) determine the association between mental disorders among critically ill patients and short-term mortality. We hypothesized that among SLE patients: 1) mental disorders are more common among critically ill patients than among those hospitalized without ICU admission; 2) ICU utilization is greater among hospitalizations with mental disorders than among those without these conditions; 3) mental disorders are associated with increased risk of short-term mortality among critically ill patients.

\section{Materials and Methods}

This was a retrospective, population-based cohort study. Because we used a publicly available, de-identified data set, the study was determined to be exempt from formal review by the Texas Tech Health Sciences Center's Institutional Review Board. This cohort study adheres to the Strengthening the Reporting of Observational Studies in Epidemiology (STROBE) reporting guidelines [18].

\section{Data sources and study population}

We used the Texas Inpatient Public Use Data File (TIPUDF) to identify the target population. In brief, the TIPUDF is an administrative data set maintained by the Texas Department of State Health Services [19] and includes inpatient discharge data from state-licensed, non-federal hospitals, and captures approximately $97 \%$ of all hospital discharges in the state.
We identified hospitalizations of patients aged $\geq 18$ years between 2009 and 2014 with a diagnosis of SLE, based on the presence of International Classification of Diseases, Ninth Revision, Clinical Modification (ICD-9-CM) code 710.0 in the principal or secondary diagnosis fields. This code was validated in administrative data, with sensitivity of $79.3 \%$, specificity of $90.2 \%$, with a positive predictive value of $95.9 \%$ [20] and was used in prior studies of SLE [21, 22]. Hospitalizations with mental disorders were identified by the presence of ICD9-CM diagnosis codes included in the Healthcare Cost and Utilization Project's Clinical Classification Software (CCS) Category 5 [23]. We excluded hospitalizations with alcohol(CCS code 660) and substance-related disorders (CCS codes 661 and 6632), as well as those with diagnoses of dementia (CCS code 653) and intellectual disability and learning disorders (CCS code 654). The last two categories generally involve multiple cognitive impairments related to medical conditions and frequently require more medical than psychiatric therapy. ICU admissions were identified based on unit-specific revenue codes for an intensive care unit or a coronary care unit. The TIPUDF dataset does not include information on the indications or specific diagnoses for admission to the ICU.

\section{Exposure and outcomes}

The primary exposure was a diagnosis of mental disorder. The primary outcome was the short-term mortality among SLE hospitalizations with mental disorders who were admitted to ICU. Short-term mortality was defined as the combination in-hospital mortality or discharge to hospice. We chose this definition of short-term mortality instead of hospital mortality because discharge to hospice is increasingly common among severely ill patients and there is a potential for underestimation of short-term mortality of severely ill populations when examined over time, if discharges to hospice are not considered [24].

The secondary outcomes included: 1) rates of ICU admission among SLE hospitalizations with and without mental disorders; 2) rates of mental disorders among SLE hospitalizations with and without ICU admission.

\section{Study variables}

Study variables were selected based on clinical plausibility and prior reports $[17,25]$. We abstracted data on patients' age, gender, race/ethnicity, health insurance, any diagnosis of mental disorders and those with a diagnosis of depression (CCS code 6572) and anxiety (CCS code 651), comorbid conditions (based on the Deyo modification of the Charlson comorbidity index [26, 27]), transfer from another facility, emergent hospital admission, admission during the weekend, type and number of organ dysfunctions [28], use of invasive mechanical ventilation (ICD-9-CM codes 96.70, 96.71, and 96.72) (termed hereafter mechanical ventilation), hemodialysis (ICD-9-CM code 39.95), hospitals' teaching status, hospital length of stay, hospital charges, hospital disposition, and year of hospitaliza- 
tion. Total hospital charges were adjusted for inflation using the consumer price index and reported as 2014 US dollars [29]. The TIPUDF and the state of Texas do not provide tools for converting hospital charges to costs.

\section{Data analysis}

We summarized categorical variables as numbers and percentages, while continuous variables were reported as mean (standard deviation (SD)) or median (interquartile range (IQR)). The chi-square test was used for group comparison involving categorical variables and Mann-Whitney test was used for comparison of continuous variables.

Because the TIPUDF dataset provides discharge-level, rather than patient-level information, precluding accounting for repeated admissions, we report the number of hospitalizations and ICU admissions as units of analysis, rather than number of patients. The state of Texas masks gender data of hospitalizations with a diagnosis of infection with the human immunodeficiency virus, and of those with ethanol or drug abuse. Thus, analyses of gender were restricted to ICU admissions where gender data were not masked.

We used logistic regression to examine the temporal trends of ICU utilization among hospitalizations with and without mental disorders.

In order to provide further anchoring context to the temporal trajectories of ICU utilization and short-term mortality, we used weighted least-squares regression to examine the corresponding temporal trends of the burden of chronic illness and illness severity, using the Deyo comorbidity index and the number of organ dysfunctions, respectively, as proxy measures.

We fit a multivariable logistic regression model to examine the association of mental disorders as independent predictor, with short-term mortality as dependent variable among ICU admissions, following examination for multicollinearity. Univariate logistic regressions were first carried out, with covariates with $\mathrm{P}<0.1$ considered for multivariate analysis. The multivariable logistic model included the following covariates: age, gender, race/ethnicity, health insurance, Deyo comorbidity index (adjusted after excluding the point score for connective tissue disease), chronic lung disease, congestive heart failure, cerebrovascular disease, chronic renal disease, diabetes, malignancy, liver disease, transfer from another hospital, number of organ failures, and year of admission. Covariates were entered using backward stepwise selection. We reported model findings as adjusted odds ratio (aOR) and $95 \%$ confidence interval (CI). In addition, we calculated the risk-adjusted probability of short-term mortality among ICU admissions with and without mental disorders, using empirical Bayesian posterior estimates from the multivariable logistic regression model. We then examined the temporal trends of risk-adjusted short-term mortality of ICU admissions with and without mental disorders using log-transformed linear regression and expressing model results as average annual percent change (AAPC) and 95\% CI.

To evaluate the robustness of the study findings on the association of mental disorders with short-term mortality among ICU admissions with SLE, two sensitivity analyses were performed, repeating separate multivariable regression modeling, employing the approach described above and substituting depression and anxiety (the most common categories of mental disorders among SLE patients [9]) for all mental disorders.

Data management was performed using Excel and Access (Microsoft, Redmond, Washington) and statistical analyses were performed with MedCalc version 19.1 (MedCalc Software, Ostend, Belgium). A two-sided P value $<0.05$ was considered statistically significant.

\section{Results}

Among 94,338 hospitalizations with SLE, 34,991 (37.1\%) were admitted to ICU. Mental disorders were reported in $35,793(37.9 \%)$ of SLE hospitalizations. The rates of ICU admission among hospitalizations with and without mental disorders were $37.0 \%$ and $37.2 \%$, respectively $(\mathrm{P}=0.5999)$. Mental disorders were present in $37.8 \%$ of all SLE admissions to ICU and in $38.0 \%$ of SLE hospitalizations without ICU admission $(\mathrm{P}=0.5408)$. The annual volumes of all SLE hospitalizations, those with and without mental disorders and of ICU admissions with and without mental disorders are summarized (Supplementary Material 1, www.jocmr.org).

\section{Cohort characteristics}

The characteristics of ICU admissions with and without mental disorders are outlined in Table 1. ICU admissions with mental disorders were more commonly aged 65 years or older ( $23.6 \%$ vs. $21.4 \%$, respectively), and more commonly white ( $46.2 \%$ vs. $33.2 \%$, respectively). ICU admissions with mental disorders had slightly higher Deyo comorbidity index (2.60 vs. 2.55 , respectively), but lower mean number of organ dysfunctions ( 0.93 vs. 1.04 , respectively). The mean hospital length of stay was slightly longer among ICU admissions with mental disorders than among those without mental disorders ( 7.3 days vs. 7.2 days, respectively), with comparable mean hospital charges ( $\$ 78,766$ vs. $\$ 80,192$, respectively).

\section{Temporal trends of ICU utilization and characteristics of ICU admissions}

The annual rate of ICU admissions among SLE hospitalizations with mental disorders increased from $36.0 \%$ to $37.4 \%$ between 2009 and 2014 (OR: 1.02/year; 95\% CI: 1.01 - 1.03; $\mathrm{P}=0.0008$ ), while remaining unchanged among those without mental disorders (OR: 1.01/year; 95\% CI: 0.99 - 1.01; P = $0.0697)$. The fraction of hospitalizations with mental disorders among ICU admissions rose from $33.1 \%$ to $42.3 \%$ between 2009 and 2014, respectively (OR: 1.09/year; 95\% CI: 1.07 $1.10 ; \mathrm{P}<0.0001)$.

The volume of ICU admissions with and without mental disorders rose between 2009 and 2014 by $60.3 \%$ vs. $7.9 \%$, respectively. As a corollary, ICU admissions with mental disorders comprised $79 \%$ of the growth in the volume of ICU admissions with SLE during the same period. 
Table 1. The Characteristics of ICU Admissions With SLE With and Without Mental Disorders

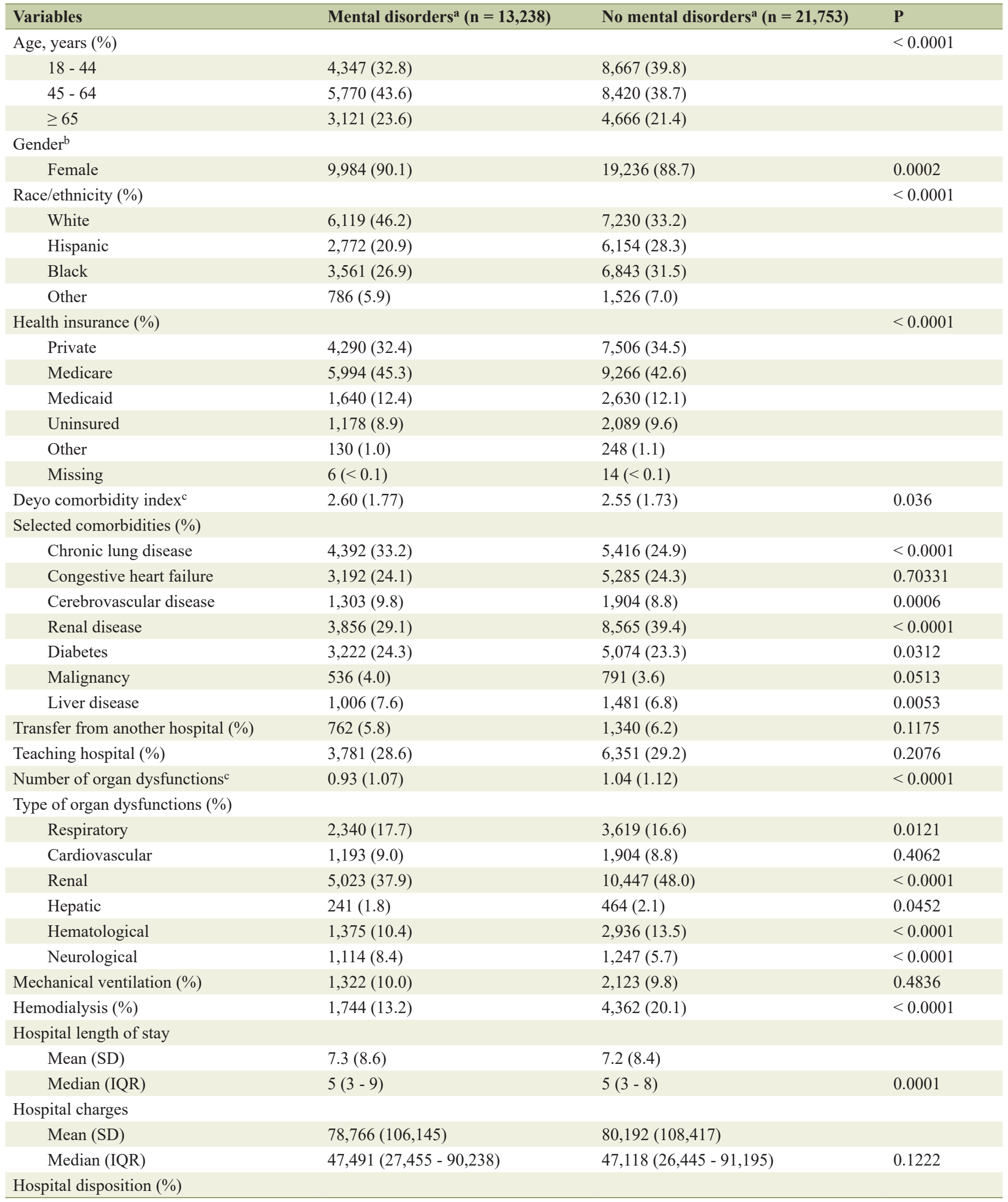


Table 1. The Characteristics of ICU Admissions With SLE With and Without Mental Disorders - (continued)

\begin{tabular}{|c|c|c|c|}
\hline Variables & Mental disorders $^{a}(n=13,238)$ & No mental disorders ${ }^{a}(n=21,753)$ & $\mathbf{P}$ \\
\hline Death & $406(3.1)$ & $990(4.6)$ & $<0.0001$ \\
\hline Hospice & $226(1.7)$ & $275(1.3)$ & 0.0008 \\
\hline Another hospital & $1,417(10.7)$ & $1,904(8.8)$ & $<0.0001$ \\
\hline Nursing facility & $1,244(9.4)$ & $1,203(5.5)$ & $<0.0001$ \\
\hline Leave against medical advice & $242(1.9)$ & $274(1.3)$ & $<0.0001$ \\
\hline
\end{tabular}

aPercentage figures may not add to 100 due to rounding. ${ }^{b}$ Gender was reported for $11,087 \mathrm{ICU}$ admissions with mental disorders and for $21,691 \mathrm{ICU}$ admissions without mental disorders; the percent figures for gender in each column refer to that column's denominator for gender. 'Mean (standard deviation (SD)). ICU: intensive care unit; SLE: systemic lupus erythematosus; IQR: interquartile range.

Table 2. Univariate and Multivariate Logistic Regression Analysis of Predictors of Short-Term Mortality Among All ICU Admissions With SLE

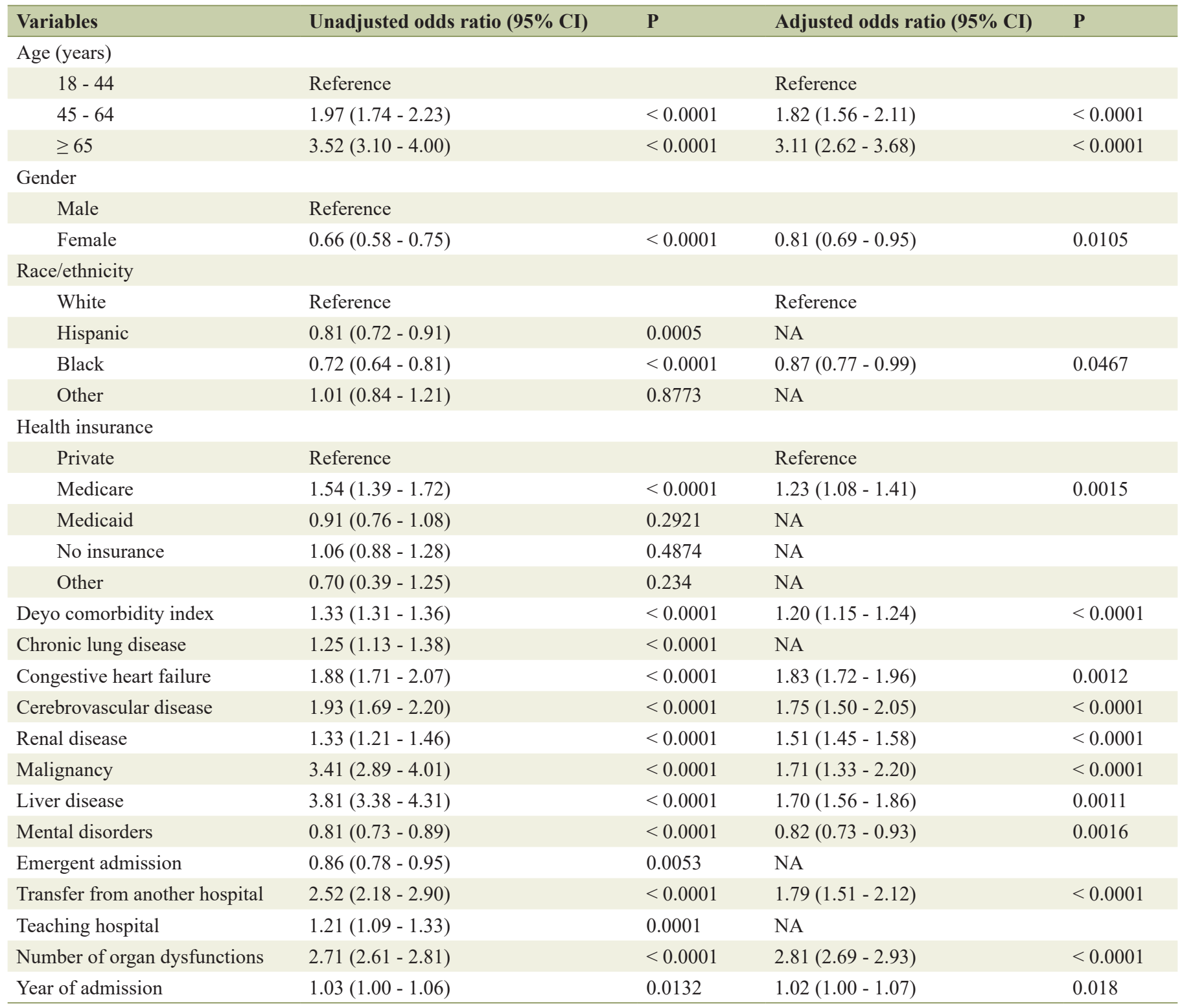

ICU: intensive care unit; SLE: systemic lupus erythematosus; 95\% CI: 95\% confidence interval. 


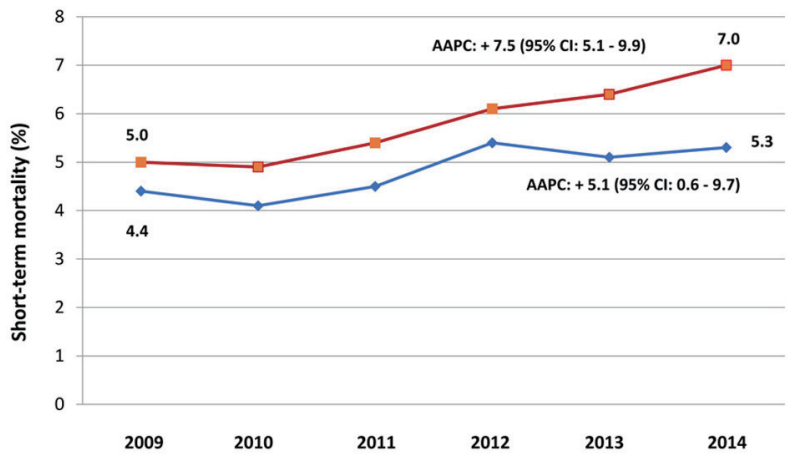

Figure 1. Temporal trends of risk-adjusted short-term mortality among ICU admissions of patients with SLE with and without mental disorders. Short-term mortality is defined as that of hospitalizations with in-hospital death or discharge to hospice. Hospitalizations with mental disorders are represented by diamond markers (blue) and those without mental disorders are represented by box markers (orange). AAPC $(95 \% \mathrm{Cl})$ denotes average annual percent change and its $95 \%$ confidence interval. Short-term mortality estimates were adjusted for age, gender, race/ethnicity, health insurance, Deyo comorbidity index, chronic lung disease, congestive heart failure, cerebrovascular disease, chronic renal disease, diabetes, malignancy, liver disease, transfer from another hospital, number of organ failures, and year of admission. ICU: intensive care unit; SLE: systemic lupus erythematosus.

Both the Deyo comorbidity index and the number of organ dysfunctions increased annually among ICU admissions with and without mental disorders (Supplementary Material 2, www.jocmr.org). The changes were modest, though all statistically significant. There was no difference between the slopes of the Deyo comorbidity index $(\mathrm{P}=0.5418)$ and the number of organ dysfunction $(\mathrm{P}=0.9696)$ between ICU admissions with and without mental disorders.

\section{The impact of mental disorders on short-term mortality among ICU admissions}

The crude short-term mortality was lower among ICU admissions with mental disorders, as compared with those without it (4.8\% vs. $5.8 \%$, respectively). On adjusted analyses, mental disorders were associated with lower odds of short-term mortality (aOR: 0.82; 95\% CI: $0.73-0.93$ ) among ICU admissions with SLE (Table 2). The adjusted probability of short-term mortality was $4.9 \%$ (95\% CI: 4.7 - 5.1) among ICU admissions with mental disorders and 5.9\% (95\% CI: 5.7 - 6.0) among ICU admissions without mental disorders.

The annual rates of risk-adjusted short-term mortality among ICU admissions with and without mental disorders are presented in Figure 1. The annual risk-adjusted short-term mortality increased over time in both groups but rose at a slower rate among ICU admissions with mental disorders (AAPC: +5.1 ; $95 \%$ CI: 0.6 - 9.7).

\section{Sensitivity analyses}

The results of sensitivity analyses are summarized (Supple- mentary Materials 3 and 4, www.jocmr.org). The findings of sensitivity analyses were consistent with the primary analyses in terms of direction and statistical significance. Both depression and anxiety were associated with markedly lower odds of short-term mortality among ICU admissions with SLE, as compared to those without these conditions.

\section{Discussion}

\section{Key findings}

In this study, the rates of mental disorders were similar among SLE hospitalizations with and without admission to the ICU. Although the rates of ICU utilization were similar among hospitalized adults with SLE with and without mental disorders, the demand for critical care resources rose over time only among the former. Mental disorders were prevalent among critically ill with SLE. However, although mental disorders were increasingly present among ICU admissions, they were associated with lower short-term mortality for the whole cohort and throughout the study period.

\section{Relationship to previous studies}

Our study represents the first examination, to our knowledge, of ICU utilization patterns among hospitalized adults with SLE and a diagnosis of mental disorders. We found, in contrast to our hypothesis, similar rates of mental disorders among SLE hospitalizations with and without ICU admission. This finding does not support an increased predisposition to critical illness by mental disorders in patients with SLE and contrasts the findings reported by Wunsch and colleagues [14]. However, the authors of the latter study have restricted their cohort to mechanically ventilated patients and defined mental disorders only as those diagnosed by a psychiatrist, though such diagnoses are often made by other physicians [30,31].

We found, unexpectedly, similar rates of ICU utilization among hospitalizations with and without mental disorders for the whole cohort. However, the rates of ICU admission increased significantly, though modestly, over time among patients with mental disorders, while remaining unchanged among those without mental disorders. The latter divergent patterns occurred despite similar trajectories over time of the burden of chronic illness and illness severity between the groups and thus cannot be attributed to reduced threshold of ICU admission for patients with mental disorders. Similarly, although the rising volume of SLE hospitalizations with mental disorders is consistent with similar patterns in the general US population [32], it would not account by itself for the rising rate of ICU admission among those with mental disorders.

A novel finding of the present study is that the growth in volume of ICU admissions over time was nearly eight times greater among SLE hospitalizations with mental disorders than among those without these conditions. As a result, mental disorders were increasingly prevalent among ICU admissions with SLE, reaching $42.3 \%$ by 2014 . Only scarce data on the 
prevalence of mental disorders among critically ill patients was reported in the general population, ranging from $6.2 \%$ among the population of mechanically ventilated patients in Denmark [14] to $36.4 \%$ in a single-center study in Singapore [15]. Only two US-based studies on mental disorders among critically ill patients were reported to date, showing mental disorders present in $25.1 \%$ [17] to $28 \%$ [33] of critically ill veterans. However, US veterans have higher rates of mental disorders as compared to the general population [34]. No prior studies have examined, to our knowledge, the temporal trends of mental disorders among ICU admissions.

The causes of the rising rate of ICU admissions among SLE hospitalizations with mental disorders and their increasingly greater representation among the critically ill are unclear and cannot be determined from administrative data. Thus, the role played by patients' mental disorders in modulating their need for ICU admission is unknown.

Mental disorders were associated with $17 \%$ lower odds of short-term mortality among critically ill patients with SLE, as compared to those without mental disorders for the whole cohort and during the study period. This favorable outcome was unexpected and occurred despite the older age and higher burden of chronic illness among critically ill patients with mental disorders, as compared to those without these conditions. Prior studies of the prognostic impact of mental disorders on short-term mortality among critically ill patients in the general population have produced mixed findings. In a population-based study of critically ill US veterans, unadjusted 30-day mortality was similar among surgical patients with and without mental disorders (3.8\% vs. $4.0 \%$, respectively), but higher among the former on adjusted analysis (OR: 1.21; 95\% CI: 1.07 - 1.37) [17]. On the other hand, in another study of veterans by the same group, involving critically ill medical patients, crude hospital mortality was lower among those with mental disorders (7.3\% vs. $8.7 \%$, respectively), but higher on adjusted analysis (OR: 1.07; 95\% CI: 1.01 - 1.14), though similar at 30-day (OR: 1.01; 95\% CI: 0.94 - 1.08) [33]. Finally, in a single-center study from the Netherlands, unadjusted hospital morality was lower among critically ill patients with mental disorders that those without this diagnosis (9.7\% vs. $13.3 \%$, respectively), but the difference was not statistically significant, likely due to the small cohort size [16].

The factors underlying the favorable association of mental disorders and short-term mortality among critically ill patients with SLE are unclear. The adverse patient- and health systemlevel factors affecting patients with mental disorders noted earlier in the general population [13] may plausibly delay recognition of health deterioration in the pre-hospital setting, and could delay patients' presentation for emergent care, resulting in greater severity of illness and thus increased risk of death. However, as noted above, the finding of similar frequency of mental disorders among SLE hospitalizations with and without ICU admission does not support increased predisposition of these patients to critical illness. Moreover, ICU admissions with mental disorders had lower number of organ dysfunctions. Finally, although depression and anxiety were reported to be among the key mental disorders associated with adverse outcomes among patients with SLE [10], both were associated with lower short-term mortality in our cohort.

In contrast to the apparently protective association of mental disorders with short-term mortality among the critically ill, they were associated with lower rates of discharge home and corresponding higher rates of transfers to other facilities. The latter findings suggest higher level of residual morbidity among critically ill SLE patients with mental disorders at the time of hospital discharge. These latter findings may be expected given the greater frequency of medical comorbid conditions among the latter group in our cohort, as well as greater physical disability among SLE patients with mental disorders. However, there have been no reports, to our knowledge, on the discharge destinations or the trajectories of health state of survivors of critical illness among SLE patients with and without mental disorders, precluding further inference.

\section{Implications of study findings}

Given the rising demand for critical care resources among hospitalized SLE patients with mental disorders, as compared to those without these conditions, further studies are needed to better understand the association between mental disorders and critical illness in this population.

Similarly, studies of more granular data are required among critically ill patients with SLE to characterize acute severity of illness, processes of care, and in-hospital clinical course of those with and without mental disorders to gain further insights into the divergent findings of the apparently "protective" association of mental disorders with short-term mortality versus its association with possibly worse residual morbidity among survivors of critical illness at the time of hospital discharge.

Finally, long-term physical, cognitive, and mental health morbidity sequelae of critical illness, currently termed post intensive care syndrome (PICS), are common among ICU survivors [35, 36]. However, no data on PICS has been reported in SLE patients. Thus, longitudinal investigations are needed to determine whether the risk of individual PICS domains and their severity differs among SLE patients surviving critical illness with and without pre-ICU diagnosis of a mental disorder.

\section{Study limitations}

Our study has several important limitations, stemming mostly from the retrospective design and use of administrative data. First, despite prior validation, there is a potential for misclassification when patient groups were selected based on ICD codes. Second, although we could not demonstrate an increased risk of critical illness among hospitalized SLE patients with mental disorders, our claims-based data cannot detect differences in the severity of mental disorders between patients who did and did not require ICU admission. Third, our data set did not include information on patients' duration of SLE, immunomodulating therapy, disease activity, and processes of care among ICU admissions with and without mental disorders, all of which may have affected our findings. Thus, although the predictive models in this study adjusted for numerous covariates, residual confounding cannot be excluded. Last, the generalizability of our findings to other states, nationally, and to other countries is unknown. 


\section{Conclusions}

The prevalence of mental disorders was high among hospitalized patients with SLE, but it did not differ among those with or without admission to ICU. Mental disorders were associated with lower short-term mortality among ICU admissions.

\section{Supplementary Material}

Suppl 1. The Annual Volume of Hospitalization and ICU Admission Among Hospitalizations With and Without Mental Disorders.

Suppl 2. Linear Regression of the Annual Changes in the Dyeo Comorbidity Index and the Number of Organ Failures Among ICU Admissions With SLE With and Without Mental Disorders.

Suppl 3. Univariate and Multivariate Logistic Regression Analysis of Predictors of Short-Term Mortality Among All ICU Admissions With SLE: The Prognostic Impact of Depression.

Suppl 4. Univariate and Multivariate Logistic Regression Analysis of Predictors of Short-Term Mortality Among All ICU Admissions With SLE: The Prognostic Impact of Anxiety.

\section{Acknowledgments}

None to declare.

\section{Financial Disclosure}

None to declare.

\section{Conflict of Interest}

None to declare.

\section{Informed Consent}

Not applicable.

\section{Author Contributions}

Lavi Oud has performed the design of work, acquisition of data, analysis and interpretation, composition, drafting, revising, editing, and final approval.

\section{Data Availability}

The data supporting the findings of this study have been deposited in the Texas Inpatient Public Use Data File, Texas De- partment of State Health Services, Center for Health Statistics, Austin, Texas, and can be accessed from http://www.dshs. state.tx.us/thcic/hospitals/Inpatientpudf.shtm.

\section{References}

1. Illescas-Montes R, Corona-Castro CC, MelguizoRodriguez L, Ruiz C, Costela-Ruiz VJ. Infectious processes and systemic lupus erythematosus. Immunology. 2019;158(3):153-160.

2. Yen EY, Shaheen M, Woo JMP, Mercer N, Li N, McCurdy DK, Karlamangla A, et al. 46-year trends in systemic lupus erythematosus mortality in the United States, 1968 to 2013: a nationwide population-based study. Ann Intern Med. 2017;167(11):777-785.

3. Jorge AM, Lu N, Zhang Y, Rai SK, Choi HK. Unchanging premature mortality trends in systemic lupus erythematosus: a general population-based study (1999-2014). Rheumatology (Oxford). 2018;57(2):337-344.

4. Pons-Estel GJ, Ugarte-Gil MF, Alarcon GS. Epidemiology of systemic lupus erythematosus. Expert Rev Clin Immunol. 2017;13(8):799-814.

5. Yazdany J, Marafino BJ, Dean ML, Bardach NS, Duseja R, Ward MM, Dudley RA. Thirty-day hospital readmissions in systemic lupus erythematosus: predictors and hospital- and state-level variation. Arthritis Rheumatol. 2014;66(10):2828-2836.

6. Quintero OL, Rojas-Villarraga A, Mantilla RD, Anaya JM. Autoimmune diseases in the intensive care unit. An update. Autoimmun Rev. 2013;12(3):380-395.

7. Hsu CL, Chen KY, Yeh PS, Hsu YL, Chang HT, Shau WY, Yu CL, et al. Outcome and prognostic factors in critically ill patients with systemic lupus erythematosus: a retrospective study. Crit Care. 2005;9(3):R177-183.

8. Zamir G, Haviv-Yadid Y, Sharif K, Bragazzi NL, Watad A, Dagan A, Amital H, et al. Mortality of patients with systemic lupus erythematosus admitted to the intensive care unit - A retrospective single-center study. Best Pract Res Clin Rheumatol. 2018;32(5):701-709.

9. Asano NM, Coriolano M, Asano BJ, Lins OG. Psychiatric comorbidities in patients with systemic lupus erythematosus: a systematic review of the last 10 years. Rev Bras Reumatol. 2013;53(5):431-437.

10. Zhang L, Fu T, Yin R, Zhang Q, Shen B. Prevalence of depression and anxiety in systemic lupus erythematosus: a systematic review and meta-analysis. BMC Psychiatry. 2017;17(1):70.

11. Walker ER, Druss BG. A public health perspective on mental and medical comorbidity. JAMA. 2016;316(10):11041105.

12. Momen NC, Plana-Ripoll O, Agerbo E, Benros ME, Borglum AD, Christensen MK, Dalsgaard S, et al. Association between mental disorders and subsequent medical conditions. N Engl J Med. 2020;382(18):1721-1731.

13. Liu NH, Daumit GL, Dua T, Aquila R, Charlson F, Cuijpers P, Druss B, et al. Excess mortality in persons with severe mental disorders: a multilevel intervention framework and priorities for clinical practice, policy and re- 
search agendas. World Psychiatry. 2017;16(1):30-40.

14. Wunsch H, Christiansen CF, Johansen MB, Olsen M, Ali $\mathrm{N}$, Angus DC, Sorensen HT. Psychiatric diagnoses and psychoactive medication use among nonsurgical critically ill patients receiving mechanical ventilation. JAMA. 2014;311(11):1133-1142.

15. Sim K, Rajasoorya C, Lam KN, Chew LS, Chan YH. High prevalence of psychiatric morbidity in a medical intensive care unit. Singapore Med J. 2001;42(11):522-525.

16. van der Kuur A, Bethlehem C, Bruins N, de Jager C, van Alst C, Haagsma OG, Keijzers A, et al. Impact of a premorbid psychiatric disorder on the incidence of delirium during ICU stay, morbidity, and long-term mortality. Crit Care Res Pract. 2019;2019:6402097.

17. Abrams TE, Vaughan-Sarrazin M, Rosenthal GE. Influence of psychiatric comorbidity on surgical mortality. Arch Surg. 2010;145(10):947-953.

18. von Elm E, Altman DG, Egger M, Pocock SJ, Gotzsche PC, Vandenbroucke JP, Initiative S. The Strengthening the Reporting of Observational Studies in Epidemiology (STROBE) statement: guidelines for reporting observational studies. PLoS Med. 2007;4(10):e296.

19. Texas inpatient public use data file. Texas Department of State Health Services, Center for Health Statistics, Austin, Texas. Available from: http://www.dshs.state.tx.us/ thcic/hospitals/Inpatientpudf.shtm.

20. Lim SS, Jamal A, Bayakly R, Tong L, Drenkard C. The Georgia Lupus Registry: accuracy of hospital discharge data in identifying systemic lupus erythematosus. Arthritis Rheum. 2007;54:S505.

21. Goss LB, Ortiz JR, Okamura DM, Hayward K, Goss CH. Significant reductions in mortality in hospitalized patients with systemic lupus erythematosus in Washington State from 2003 to 2011. PLoS One. 2015;10(6):e0128920.

22. Murray SG, Schmajuk G, Trupin L, Gensler L, Katz PP, Yelin EH, Gansky SA, et al. National lupus hospitalization trends reveal rising rates of herpes zoster and declines in pneumocystis pneumonia. PLoS One. 2016;11(1):e0144918.

23. Clinical Classification Software (CCS) for ICD-9-CM. Healthcare Cost and Utilization Project. Available from: https://www.hcup-us.ahrq.gov/toolssoftware/ccs/ccs.jsp.

24. Rhee C, Dantes R, Epstein L, Murphy DJ, Seymour CW, Iwashyna TJ, Kadri SS, et al. Incidence and Trends of Sepsis in US Hospitals Using Clinical vs Claims Data,
2009-2014. JAMA. 2017;318(13):1241-1249.

25. Vincent JL, Lefrant JY, Kotfis K, Nanchal R, MartinLoeches I, Wittebole X, Sakka SG, et al. Comparison of European ICU patients in 2012 (ICON) versus 2002 (SOAP). Intensive Care Med. 2018;44(3):337-344.

26. Quan H, Sundararajan V, Halfon P, Fong A, Burnand B, Luthi JC, Saunders LD, et al. Coding algorithms for defining comorbidities in ICD-9-CM and ICD-10 administrative data. Med Care. 2005;43(11):1130-1139.

27. Quan H, Li B, Couris CM, Fushimi K, Graham P, Hider $\mathrm{P}$, Januel JM, et al. Updating and validating the Charlson comorbidity index and score for risk adjustment in hospital discharge abstracts using data from 6 countries. Am J Epidemiol. 2011;173(6):676-682.

28. Martin GS, Mannino DM, Eaton S, Moss M. The epidemiology of sepsis in the United States from 1979 through 2000. N Engl J Med. 2003;348(16):1546-1554.

29. Bureau of Labor Statistics. United States Department of Labor. 2019. Available from: http://www.bls.gov/bls/inflation.htm.

30. Verhaak PF. Analysis of referrals of mental health problems by general practitioners. Br J Gen Pract. 1993;43(370):203-208.

31. Parikh SV, Lin E, Lesage AD. Mental health treatment in Ontario: selected comparisons between the primary care and specialty sectors. Can J Psychiatry. 1997;42(9):929934.

32. Heslin KC, Elixhauser A, Steiner CA. Hospitalizations involving mental and substance use disorders among adults, 2012: Statistical Brief\#191. In: Healthcare Cost and Utilization Project (HCUP) Statistical Briefs. Rockville (MD), 2006.

33. Abrams TE, Vaughan-Sarrazin M, Rosenthal GE. Preexisting comorbid psychiatric conditions and mortality in nonsurgical intensive care patients. Am J Crit Care. 2010;19(3):241-249.

34. Olenick M, Flowers M, Diaz VJ. US veterans and their unique issues: enhancing health care professional awareness. Adv Med Educ Pract. 2015;6:635-639.

35. Rawal G, Yadav S, Kumar R. Post-intensive care syndrome: an overview. J Transl Int Med. 2017;5(2):90-92.

36. Inoue $\mathrm{S}$, Hatakeyama J, Kondo Y, Hifumi T, Sakuramoto $\mathrm{H}$, Kawasaki T, Taito S, et al. Post-intensive care syndrome: its pathophysiology, prevention, and future directions. Acute Med Surg. 2019;6(3):233-246. 